\title{
Estimation of malaria transmission intensity in Sennar state, central Sudan
}

\author{
Z.A. Elmahdi, ${ }^{1}$ A.A. Nugud ${ }^{2}$ and I.M. Elhassan ${ }^{3}$
}

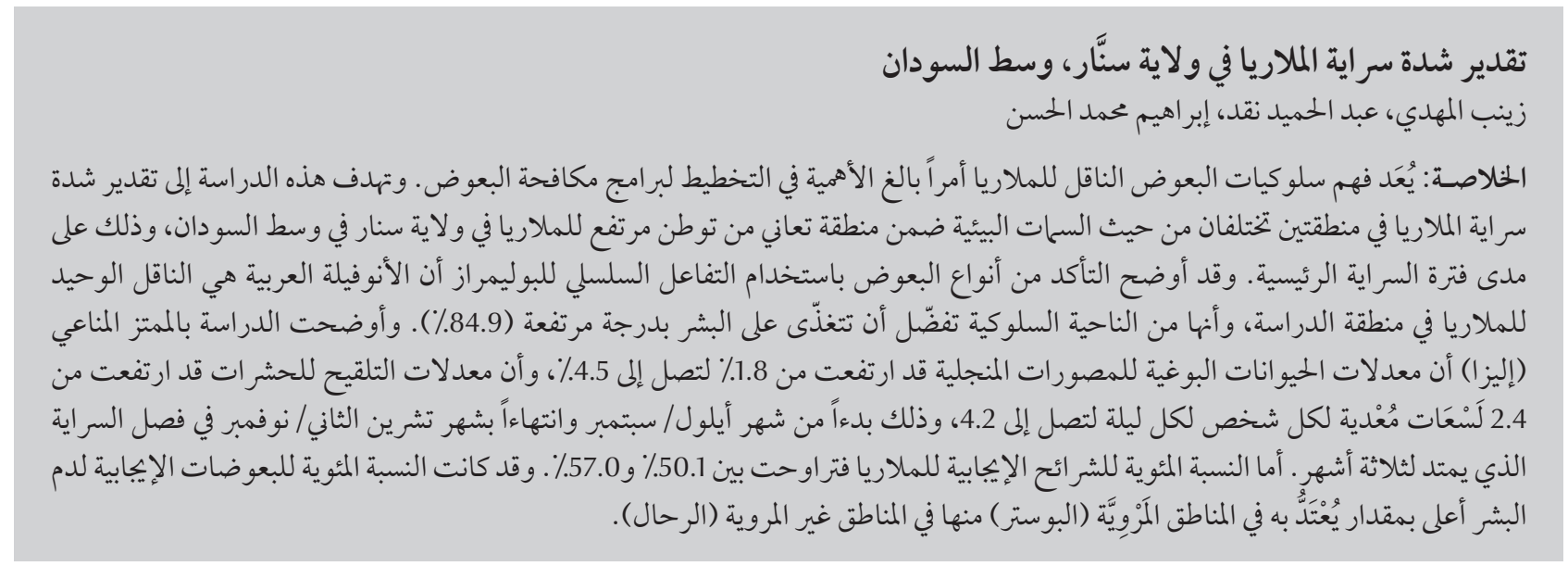

ABSTRACT Understanding the behaviour of malaria vectors is crucial for planning mosquito control programmes. The aim of this study was to estimate the malaria transmission intensity in 2 different ecological zones in a highly endemic malaria area of Sennar state in central Sudan over the main transmission period. Species confirmation by PCR indicated that Anopheles arabiensis was the only malaria vector in the study area, with high anthropophilic behaviour (84.9\% human-feeding). ELISA studies showed Plasmodium falciparum sporozoite rates rose from $1.8 \%$ to $4.5 \%$ and the average entomological inoculation rates rose from 2.4 to 4.2 infectious bites per person per night in September (the beginning) to November (the end) of the 3-month transmission season. The proportion of malaria-positive slides ranged from $50.1 \%$ to $57.0 \%$. The proportion of human-blood positive mosquitoes was significantly higher in the irrigated area (El Booster) compared with the non-irrigated area (Rahal).

\section{Estimation de l'intensité de la transmission du paludisme dans l'État de Sennar (centre du Soudan)}

RÉSUMÉ La compréhension du comportement des vecteurs du paludisme est cruciale pour la planification des programmes de lutte contre ces moustiques. L'objectif de la présente étude était d'estimer, pendant la principale période de transmission, l'intensité de la transmission du paludisme dans deux zones écologiquement différentes de l'état de Sennar, au centre du Soudan, où le paludisme est fortement endémique. La confirmation de l'espèce par la méthode PCR a indiqué qu'Anopheles arabiensis était le seul vecteur du paludisme présent dans la zone étudiée, et qu'il avait un comportement hautement anthropophile (alimentation d'origine humaine à $84,9 \%$ ). La méthode ELISA a révélé que les taux sporozoïtiques de Plasmodium falciparum avaient augmenté de 1,8 \% à 4,5\% et les taux d'inoculation entomologique moyens de 2,4 à 4,2 piqûres infectieuses par personne et par nuit entre septembre, le début de la période, et novembre, c'est-à-dire la fin de la saison de transmission qui dure trois mois. La proportion de lames positives pour le paludisme allait de 50,1 \% à 57,0 \%. La proportion de moustiques positifs pour le sang humain était significativement plus elevée dans la zone irriguée (El Booster) que dans la zone non irriguée (Rahal). 


\section{Introduction}

Malaria is a major health problem in Sudan. With the exception of a small area at the Sudan-Egypt border, the whole population is considered to be at risk of infection. More than $95 \%$ of malaria cases in Sudan are due to Plasmodium falciparum [1] and mosquitoes of the Anopheles gambiae complex Giles are the main vectors [2-6]. As malaria transmission in Africa is characterized by complex species heterogeneity, understanding the factors that facilitate transmission are very important in targeted malaria control and eradication programmes.

The sporozoite infection rate (SR) of the mosquito population is a powerful and sensitive measure for describing the epidemiology of malaria in a particular area. It is also used to determine the entomological inoculation rate (EIR), to establish both vector identity and differences in transmission intensity over space and time and for the assessment of malaria control programmes. The EIR estimates the level of exposure to $P$. falciparum-infected mosquitoes. It is the most common measure for assessing malaria endemicity and transmission intensity and is particularly useful when estimating the effect of human-vector contact [7]. SR and EIR are largely dependent on environmental conditions and would be expected to vary seasonally. Studies have compared transmission intensity in different ecological zones and have reported significant variations in the transmission intensity of malaria between rural and urban settings within the same locality and even between villages separated by short distances [8]. Development of enzyme-linked immunosorbent assays (ELISA) for the detection of circumsporozoite protein provide an effective tool for determination of the infection rates in mosquitoes and the consequent incrimination of malaria vector(s) [9] and for identifying the blood meals of vectors [10]. Understanding the feeding preferences of a given malaria vector has paramount epidemiological importance in selection of the appropriate vector control measure(s).

In the present study, we used polymerase chain reaction (PCR) for the identification of the vector responsible for malaria transmission and ELISA to determine the SR and blood-meal sources of the malaria vectors in an area of central Sudan characterized by highly seasonal malaria transmission.

\section{Methods}

\section{Study area}

The study was conducted in Sennar state which lies in a rich savannah region between latitude $12.5^{\circ}-14.7^{\circ} \mathrm{N}$ and longitude $32.9^{\circ}-35.4^{\circ} \mathrm{S}$ in central eastern Sudan. It shares borders with Gezira state in the north, White Nile and Upper Nile states in the west, Gadarif state in the east and Blue Nile state and Ethiopia in the south. The summer extends from March to May, with average daily temperatures of $32-40^{\circ} \mathrm{C}$ and relative humidity of $25 \%$. The rainy season starts early in June and continues until September. Winter starts in October with average daily temperatures of $20-25^{\circ} \mathrm{C}$ [Sudan Meteorological Services, 2005, unpublished].

The population is close to 2 million. The study area is characterized by stable and highly seasonal malaria transmission. For the purpose of this study the following sites were selected:

- Rahal area $\left(33^{\circ} 55^{\prime}\right.$ E, $\left.13^{\circ} 10^{\prime} \mathrm{N}\right)$; lies in central-east Sennar and it is an urban area. Most of the people work as employees in different organizations or have their own jobs. The houses are generally well-constructed. Around $90 \%$ of the people, especially children, use impregnated or non-impregnated bednets. The main mosquito breeding sites are rain pools.

- El Booster area in western Sennar $\left(33^{\circ} 36^{\prime} \mathrm{E}, 13^{\circ} 32^{\prime} \mathrm{N}\right)$. It lies in the middle of cotton and sugarcane agricultural schemes. Most of the people are working in farming in cotton and sugar schemes. The houses are poorly constructed and the use of bednets is not common. The breeding sites are mainly rain pools and different types of drains.

Ethical approval for this project was obtained from the national ethics committee and the ethics committee of the Institute of Endemic Diseases, University of Khartoum.

\section{Mosquito sampling and processing}

\section{Mosquito collections}

Entomological surveys were conducted during the main malaria transmission months between September and November 2008. Mosquito collections were made once per month from each site from 7 randomly selected rooms by using the pyrethrum spray catch method. Mosquitoes were sorted according to their feeding status as unfed, fresh fed, half-gravid and gravid and then identified to species based on morphological characteristics [11]. Specimens resembling An. gambiae were individually preserved in labelled tubes containing silica gel and stored at room temperature until further processing.

\section{Species identifications using PCR}

Females belonging to the An. gambiae complex were identified to species using the PCR technique described by Scott et al. [12]. A small segment from a leg of a mosquito was placed directly into reaction mixture containing speciesspecific primers, dNTPs, buffer and polymerase. The resultant amplicon was detected on $2 \%$ agarose gel stained with ethidium bromide.

\section{Detection of sporozoite infections}

For detection of sporozoite infections, circumsporozoite ELISA was carried out as previously described $[9,13]$. In brief, the head and thorax of each mosquito were separated from the abdomen and tested for the presence of $P$. 
falciparum circumsporozoite antigen. Mosquitoes were ground in $50 \mu \mathrm{L}$ of boiled casein containing (Igepal CA 630) and the final volume was brought to $250 \mu \mathrm{L}$ with blocking buffer; $50 \mu \mathrm{L}$ of the triturate was used in sporozoite enzyme-linked immunosorbent assays. Positive reactions were determined visually and spectrophotometrically. All ELISA chemicals were obtained from Sigma and the reagents (MAPs and peroxidases) were provided by the US Centers for Disease Control and Prevention and Kirkegaard and Perry Laboratories Inc.

\section{Identification of blood-meal sources}

All blood-fed An. arabiensis were tested against anti-human antigens using the ELISA method of Bier et al. [14]. Mosquito abdomens were ground in 50 $\mu \mathrm{L}$ grinding buffer and completed the total volume to $450 \mu \mathrm{L} ; 50 \mu \mathrm{L}$ of mosquito homogenate was used for blood meal detection. Positive samples were determined visually and spectrophotometrically.

\section{Calculation of SR and EIR}

SR was calculated by dividing the number of mosquitoes positive for P. falciparum by the number of tested mosquitoes. The EIR, expressed as the number of infectious bites per person per unit time, was derived as a product of the SR and the human biting rate [15]. The monthly EIRs were derived by multiplying the daily EIR [infectious bites per person per night $(\mathrm{ib} / \mathrm{p} / \mathrm{n})]$ by 30 days.

\section{Parasitological data}

Parasitological data on the prevalence of malaria in the area were obtained from the malaria health authority's reports (State Ministry of Health, central Sudan). These data were used to correlate entomological parameters with the prevalence of malaria in the study area.

\section{Statistical analysis}

Data entry was performed using the Microsoft Excel program. SPSS, version 11.5 software was used for data analysis. Frequency distributions were calculated. Student $t$-test was used to evaluate whether there was a significant difference between measures from the 2 areas. The level of $P<0.05$ was used to indicate statistical significance.

\section{Results}

\section{Vector identification}

A total of 970 anopheline mosquitoes were collected. Morphological identification and subsequent application of species-specific PCR analysis confirmed that An. arabiensis was the only member of the An. gambiae complex in the study area. Out of 412 mosquitoes (randomly selected) 370 (89.8\%) were identified as An. arabiensis.

\section{Sporozoite infection rate}

All An. arabiensis were tested for the presence of P. falciparum sporozoite and 30 specimens were found to be positive. The mean positivity rates over the 3-month period were $4.2 \%$ (SD 2.0\%) and 2.3\% (SD 0.8\%) in El Booster and Rahal areas respectively $(P>0.05)$. The SR values increased from September through to November in each area (Table 1) and both areas combined (Figure 1).

\section{Blood-meal sources}

A total of 360 An. arabiensis collected from the study sites were tested for human blood and 307 mosquitoes (85.3\%) were positive for human blood. The proportion of mosquitoes that were human-blood positive was significantly higher in El Booster (86.9\%) than in Rahal (82.9\%) $(P=0.01)$.

\section{Entomological inoculation rate}

The mean EIR over the 3-month transmission season was significantly higher in El Booster [5.6 (SD 2.0) $\mathrm{ib} / \mathrm{p} / \mathrm{n}$ ] than Rahal [2.1 (SD 0.5) $\mathrm{ib} / \mathrm{p} / \mathrm{n}](P=0.01)$. The EIR in the 2 areas combined increased from September through to November (Figure 1). The highest EIR values were recorded in November in each area: 6.8 and $2.5 \mathrm{ib} / \mathrm{p} / \mathrm{n}$ for El Booster and Rahal respectively.

\begin{tabular}{|c|c|c|c|c|}
\hline \multirow[t]{2}{*}{ Measure/area } & \multicolumn{3}{|c|}{ Month } & \multirow[t]{2}{*}{ Mean (SD) } \\
\hline & Sep. & Oct. & Nov. & \\
\hline \multicolumn{5}{|c|}{ Sporozoite rate (\%) } \\
\hline El Booster & 2.1 & 4.4 & 6.0 & $4.2(2.0)$ \\
\hline Rahal & 1.4 & 2.5 & 3.0 & $2.3(0.8)^{\mathrm{a}}$ \\
\hline \multicolumn{5}{|c|}{$\begin{array}{l}\text { Entomological inoculation rate (infectious } \\
\text { bites/person/night) }\end{array}$} \\
\hline El Booster & 3.3 & 6.6 & 6.8 & $5.6(2.0)$ \\
\hline Rahal & 1.5 & 2.3 & 2.5 & $2.1(0.5)^{\mathrm{b}}$ \\
\hline
\end{tabular}

${ }^{a} P>0.05 ;{ }^{b} P=0.01$ between the 2 areas.

$S D=$ standard deviation 


\section{Relationship between parasitological and entomological parameters}

Theproportion of malaria-positive slides rose from 50.1\% in September through to $57.0 \%$ in November. High positive correlations were found between the proportion of malaria-positive slides and both SR (0.88) and EIR (0.90) (Figure 1).

\section{Discussion}

Information concerning the species composition, biology and behaviour of malaria vectors is crucial for understanding the vector's epidemiological role in malaria transmission and therefore for designing and planning control programmes. Previously, vector identification in Sudan was conducted through morphological studies which indicated that the principal vector of malaria in the country was An. gambiae s.l. The subsequent genetic studies (crossing experiments) revealed that the only member of An. gambie found in central Sudan was An. gambiae species B $[16,17]$. This was followed by cytotaxonomy studies which showed that An. arabiensis was the only malaria vector in central

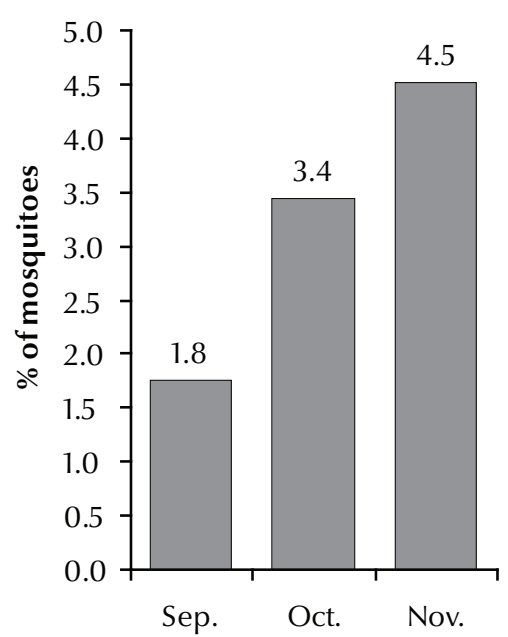

(a) Sporozoite rate
Sudan [5]. The non-extracted DNA protocol of Scott et al. has been shown to be sensitive [12], easy and suitable for routine work as large number of samples can be identified in a single day [18]. In the present study, we used the PCR technique in order to confirm the species identification carried out earlier in Sudan. Our results confirmed that $A n$. arabiensis was the only member of An. gambiae complex present in the study areas.

In this study we found that the SR ranged from $1.4 \%$ to $6.0 \%$. The highest rates were reported during the peak of the transmission season (November) in both sites, when the conditions are less favourable for breeding and the vector population is mostly composed of older mosquitoes [19]. The infectivity rates in both study sites were found to be within the normal range reported for members of An gambiae complex [20]. Increases in SR were observed from the beginning of the transmission season (September) to the peak of the rainy season (November) in both areas. A relatively broad range of SRs have been recorded in other African countries [21-23]. In central Sudan, no sporozoites were detected in all dissections carried out in the Blue Nile health project area which covered the Gezira irrigation in the period between 1981 and 1989. However, Elsafi reported a SR of $0.45 \%$ in Gezira area of central Sudan in 1992 [unpublished data], while in the Gadaref area of eastern Sudan, a SR of $1.44 \%$ was reported during the malaria transmission season of 1995 [6]. The relatively high SRs recorded in the present study could be explained the high anthropophilic feeding behaviour of An. arabiensis in the absence of other animals. In addition, all samples of the current study were collected during the malaria transmission season.

Malaria transmission intensity is best expressed as the EIR, which directly reflects the exposure of humans to pathogenic Plasmodium spp. parasites [24]. It has been observed that EIR values in Africa vary widely both spatially and temporally from 0 to 884 infectious bites per person per year [25], with rural areas experiencing higher intensities of transmission than urban areas [24]. In the present study we compared 2 sites representing rural and irrigated areas in order to get more accurate information on variations in the transmission of malaria in different environmental and ecological situations of this region. Irrigation schemes provide ideal breeding

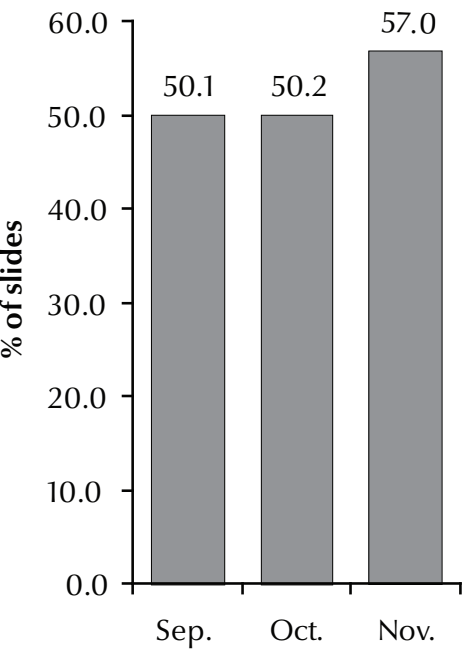

(c) Malaria-positive slides rate

Figure 1 Monthly sporozoite rate, entomological inoculation rate and proportion of malaria-positive slides in the 2 study areas of Sennar state, central Sudan (combined data) 
sites for malaria vectors in Africa [26]. However, no simple association has been found between irrigation and the degree of exposure to malaria parasites (EIR) as measured using classical entomological methods $[27,28]$. Our results indicated that exposure to malaria infection was significantly higher in the study population of the irrigated area (El Booster) compared with the non-irrigated area (Rahal). This result is in agreement with a previously study carried out in Ghana [28].

A number of studies have reported that the high EIR values and therefore high risk of exposure to infective bites usually occur during the wet seasons in which $75 \%$ of the annual EIR is recorded [24]. Our results showed that the overall EIR ranged between 1.5-6.9 $\mathrm{ib} / \mathrm{p} / \mathrm{n}$. These values were comparable to other EIR values reported from different African countries,; for example, in Kenya Shililu et al. reported a range of $0-7 \mathrm{ib} / \mathrm{p} / \mathrm{ts}$ [29]. Furthermore, ranges of $3-5 \mathrm{ib} / \mathrm{p} / \mathrm{ts}$ and $5-12 \mathrm{ib} / \mathrm{p} / \mathrm{ts}$ were reported from Gambia and Burundi respectively [30,31]. Nonetheless the highest ranges of EIR (122-702 ib/p/ ts) have been recorded from Tanzania [26,32].

We noted an increase in the risk of malaria infection from the beginning of the malaria transmission season (September) through to the end of the malaria season (November), as indicated by the increases in SR and EIR values. A significant positive correlation was found between the SR, EIR and the proportions of malaria-positive slides reported during the study months.
Identification of blood-meal sources for mosquitoes is an important factor in the study of malaria transmission and vectorial capacity [33]. Also feeding behaviour is of great importance with respect to the planning of vector control programmes [34]. Our overall results of blood-meal analysis showed that the main source of blood for the majority (almost $85 \%$ ) of An. arabiensis was human. Generally, An. arabiensis is regarded as being more exophilic, exophagic and zoophilic than An. gambiae s.s., but although it is a highly efficient vector of malaria [34], it appears to be an east-west behavioural cline. For $A n$. arabiensis populations in West Africa, the proportion of blood meals from humans has been reported as 80\%-100\% indoors, with most feeding and resting occurring indoors [35]. By contrast, in East Africa, a greater proportion of $A n$. arabiensis feed on cattle and rest outdoors. In Tanzania, for example, White et al. reported human blood indices of $61 \%$ and $7 \%$ for samples collected indoors and outdoors, respectively [36]. These behavioural differences may be linked to observed genetic variability [37] and the opportunistic behaviour of An. arabiensis [38]. However, the high rate of feeding on humans in this study showed that humans were indeed the preferred host, and $A n$. arabiensis is highly anthropophilic. These results confirmed a previously published report from Ethiopia [39]. In the current study the proportion of human-blood positive mosquitoes was significantly higher in El Booster compared with Rahal. This could be explained by the fact that inhabitants of Rahal area tend to keep cattle and other grazing animals around their settlements and these animals may have a zooprophylactic effect. In this study, the high percentage of indoor collected An. arabiensis females from the 2 areas were found to be fed on humans. Thus, the use of long-lasting insecticidal nets and residual spraying are the most suitable measures for malaria control in central Sudan.

\section{Conclusions}

This study confirmed that An. arabiensis is the only member of An. gambiae complex present in Elbooster and Rahal areas of Sennar state. Malaria in central Sudan is highly seasonal with the peak during the known transmission season occurring in November.

\section{Acknowledgements}

The technical assistance of staff of the Malaria Training Centre, Sennarstate, is greatly acknowledged. Dr Robert Wirtz and Dr Melissa Avery, Department of Medical Entomology, Centre of Disease Control and Prevention, Atlanta, United States of America, are greatly acknowledged for providing ELISA reagents. Dr M. Balkew, University of Addis Ababa, is thanked for his technical advice and valuable comments. This study obtained financial assistance from the MIM/TDR research project (ID No. TDA 40048A).

\section{References}

1. Elhassan IM et al. High proportion of subclinical Plasmodium falciparum infections in an area of seasonal and unstable malaria in Sudan. American Journal of Tropical Medicine and $\mathrm{Hy}$ giene, 1995, 53:78-83.

2. Zahar AR. Vector bionomics in the epidemiology and control of malaria. Part 1. Section $\amalg,(D)$ East Africa. Geneva, World Health Organization, 1985 (WHO/DOCVBC/85.3).

3. Lewis DJ. Some mosquitoes of the Blue Nile valley in Republic of the Sudan. Bulletin of Entomological Research, 1958, 49:133-155.
4. Haridi AM. Partial exophily of Anopheles gambiae species B in the Khashm Elgirba area in eastern Sudan. Bulletin of the World Health Organization, 1972, 46:39-46.

5. Petrarca V et al. Cytogenetics of the Anopheles gambiae complex in Sudan, with special reference to An. arabiensis: relationships with East and West African populations. Medical and Veterinary Entomology, 2000, 14:149-164.

6. Karrar AA. Seasonality of malaria transmission in Assar and Daraweesh villages (Gadaref Province, Gadaref, State, Eastern 
Sudan [MSc thesis]. Khartoum, Sudan, Department of Zoology, University of Khartoum, 1996.

7. Kelly-Hope LA, McKenzie FE. The multiplicity of malaria transmission: a review of entomological inoculation rate measurements and methods across sub-Saharan Africa. Malaria Journal, 2009, 8:19.

8. Robert $\mathrm{V}$ et al. Malaria transmission in urban sub-Saharan Africa. American Journal of Tropical Medicine and Hygiene, 2003, 68:169-176.

9. Wirtz RA et al. Field evaluation of enzyme-linked immunosorbent assays for Plasmodium falciparum and Plasmodium vivax sporozoites in mosquitoes (Diptera: Culicidae) from Papua New Guinea. Journal of Medical Entomology, 1987, 24:433-437.

10. Voller A et al. Enzyme-linked immunosorbent assay. In: Rose NR, Fredman H, eds. Manual of clinical immunology, 2nd ed. Washington DC, American Society of Microbiology, 1980:359371.

11. Gillies MT et al. The Anophelinae of Africa south of Sahara (Ethiopian zoogeografical region). Johannesburg, South African Institute of Medical Research, 1968 (Publication No. 5).

12. Scott JA, Brogdon WG, Collins FH. Identification of single specimens of the Anopheles gambiae complex by the polymerase chain reaction. American Journal of Tropical Medicine and Hygiene, 1993, 49:520-529.

13. Zavala F et al. Monoclonal antibodies to circumsporozoite proteins identify the species of malaria parasite in infected mosquitoes. Nature, 1982, 299:737-738.

14. Beier JC et al. Bloodmeal identification by direct enzymelinked immunosorbent assay (ELISA), tested on Anopheles (Diptera: Culicidae) in Kenya. Journal of Medical Entomology, 1988, 25:9-16.

15. MacDonald G. The epidemiology and control of malaria. Oxford, Oxford University Press, 1957.

16. Lewis DJ. Some mosquitos of the Sudan. Bulletin of Entomological Research, 1956, 47:723-735.

17. Petrarca $V$ et al. Dati preliminary sul complesso Anopheles gambiae in Sudan. Parassitologia, 1986, 28:304-306.

18. Van Rensburg AJ et al. The polymerase chain reaction method as a tool for identifying members of the Anopheles gambiae complex (Diptera:Culicidae) in northeastern Tanzania. Journal of the American Mosquito Control Association, 1996, 12:271-274.

19. Beier JC. Malaria parasite development in mosquitoes. Annals Review of Entomology, 1998, 43:519-543.

20. Magesa SM et al. Trial of pyrethroid impregnated bednets in an area of Tanzania holoendemic for malaria. Part 2. Effects on the malaria vector population. Acta Tropica, 1991, 49:97-108.

21. Joshi GP, Service MW, Pradhan GD. A survey of species A and B of the Anopheles gambiae Giles complex in the Kisumu area of Kenya prior to insecticidal spraying with OMS-43 (fenitrothion). Annals of Tropical Medicine and Parasitology, 1975, 69:91-104.

22. Fettene $\mathrm{M}$ et al. Behaviour of Anopheles arabiensis and Anopheles quadriannulatus sp. B mosquitoes and malaria transmission and Southwestern Ethiopia. Africa Entomology, 2004, 12:83-87.

23. Okbaldet YB. Biology and transmission potential of malaria vector moasquitoes in Elabered sub-zone, Eritrea [MSc thesis]. Bloemfontein, South Africa, Department of Zoology and Entomology, Faculty of Natural and Agricultural Sciences, University of Free State, 2001.
24. Hay SI et al. Annual Plasmodium falciparum entomological inoculation rates (EIR) across Africa: literature survey, Internet access and review. Transactions of the Royal Society of Tropical Medicine and Hygiene, 2000, 94:113-127.

25. Mbogo $\mathrm{CN}$ et al. Relationships between Plasmodium falciparum transmission by vector populations and the incidence of severe disease at nine sites on the Kenyan coast. American Journal of Tropical Medicine and Hygiene, 1995, 52:201-206.

26. Temu EA et al. The role of four anopheline species (Diptera: Culicidae) in malaria transmission in coastal Tanzania. Transactions of the Royal Society of Tropical Medicine and Hygiene, 1998, 92:152-158.

27. Ijumba JN et al. Irrigated crop production is associated with less malaria than traditional agricultural practices in Tanzania. Transactions of the Royal Society of Tropical Medicine and $\mathrm{Hy}$ giene, 2002, 96:476-480.

28. Appawu M et al. Malaria transmission dynamics at a site in northern Ghana proposed for testing malaria vaccines. Tropical Medicine and International Health, 2004, 9:164-170.

29. Shililu Jl et al. Seasonal density, SRs and entomological inoculation rates of Anopheles gambiae and Anopheles funestus in highaltitude sugarcane growing zones in Western Kenya. Topical Medicine and International Health, 1998, 3(9):706-710.

30. Lindsay SW et al. A malaria control trial using insecticide-treated bed nets and targeted chemoprophylaxis in a rural area of The Gambia, west Africa. 7. Impact of permethrin-impregnated bed nets on malaria vectors. Transactions of the Royal Society of Tropical Medicine and Hygiene, 1993, 87(Suppl. 2):45-51.

31. Van Bortel W et al. Deltamethrin-impregnated bednets as an operational tool for malaria control in a hyper-endemic region of Burundi: impact on vector population and malaria morbidity. Tropical Medicine \& International Health, 1996, 1:824-835.

32. Shiff CJ et al. Malaria infection potential of anopheline mosquitoes sampled by light trapping indoors in coastal Tanzanian villages. Medical and Veterinary Entomology, 1995, 9:256-262.

33. Hewitt S, Rowland M. Control of zoophilic malaria vectors by applying pyrethroid insecticides to cattle. Tropical Medicine and International Health, 1999, 4:481-486.

34. White GB. Anopheles gambiae complex and disease transmission in Africa. Transactions of the Royal Society of Tropical Medicine and Hygiene, 1974, 68:278-301.

35. Molineaux L, Gramiccia G. The Garki project. Geneva, World Health Organization, 1980:109-115.

36. White GB. The Anopheles gambiae complex and malaria transmission around Kisumu, Kenya. Transactions of the Royal Society of Tropical Medicine and Hygiene, 1972, 66:572-581.

37. Coluzzi $\mathrm{M}$ et al. Chromosomal differentiation and adaptation to human environments in the Anopheles gambiae complex. Transactions of the Royal Society of Tropical Medicine and Hygiene, 1979, 73:483-497.

38. Gillies MT, Coetzee M. A supplement to the anopheline of Africa South of the Sahara. Johannesburg, South African Institute of Medical Research, 1987 (Publication No. 55).

39. Tirados I et al. Blood-feeding behaviour of the malarial mosquito Anopheles arabiensis: implications for vector control. Medical and Veterinary Entomology, 2006, 20:425-437. 